\title{
Jules Verne - a new polishing technique related to FJP
}

\author{
Silvia M. Booij*a, Oliver W. Fähnleb, Mark Meeder ${ }^{\mathrm{b}}$, Torsten Wons ${ }^{\mathrm{b}}$, and Joseph J.M. Braata \\ *s.m.booij@tnw.tudelft.nl \\ aOptics Research Group, Delft Univ. of Technology, Lorentzweg 1, 2628 CJ Delft, the Netherlands \\ bFISBA-Optik, Rorschacherstrasse 268, CH-9016 St. Gallen, Switzerland
}

\begin{abstract}
A variation on the fluid jet polishing (FJP) technique, arbitrarily named Jules Verne (JV), will be described in this article. Jules Verne is a glass processing technique that removes material due to the fact that the tool and the surface are in close contact, and a slurry moves in between the tool and the surface. This approach has both advantages and disadvantages with respect to the original FJP modus: it enables a feed-controlled machining process, but deeper lying areas are harder to reach. A simulation model will be presented that predicts the flow of the slurry in the Jules Verne setup, which is followed by the computation of the trajectories of the particles in the flow. Furthermore, experimental data will be reported demonstrating the feasibility of the JV idea. A model will also be presented simulating the interaction between the surface and the impinging abrasives at a microscopic level, enabling the prediction of the final surface roughness.
\end{abstract}

Keywords: polishing, shaping, abrasive fluid jet, roughness

\section{INTRODUCTION}

This article will cover the novel idea of polishing with the so called Jules Verne technique, a new polishing technique that is related to Fluid Jet Polishing (FJP) [1]. Similar to PACE (plasma assisted chemical etching), a small cup-wheel like tool is positioned within a Computer Numerically Controlled (CNC) machine close to the surface to be machined. An abrasive slurry is fed into the cup wheel along its axis of symmetry. If the gap between tool and workpiece is small enough, pressure is built up within the cup wheel and material removal is achieved along the circular edge of the rotating cup wheel. The advantage of this approach is that it enables a feed-controlled machining process. This article will cover the experimental setup that has been built on a computer numerically controlled machine (Section 2), a simulation of the flow of the slurry will be treated in Section 3, the results of some initial experiments will be described in Section 4, and some issues concerning the final roughness of the treated surface will be covered in Section 5.

\section{JULES VERNE}

\subsection{The setup}

The new method for shaping, shape corrections and polishing that has been invented and that is the subject of this article is arbitrarily named Jules Verne (JV). The new method works with abrasives in water, just like in the case of FJP, but it removes material by moving abrasive particles over the surface in a fashion comparable to that in the case of PACE (plasma assisted chemical etching). A small cup-wheel like tool is positioned within a CNC machine close to the surface to be machined. An abrasive slurry is fed into the cup wheel along its axis of symmetry. If the gap between tool and workpiece is small enough (typical stand-off distance of $50 \mu \mathrm{m}$ ), pressure is built up within the cup wheel and material removal is achieved along the circular edge of the rotating cup wheel. The velocity in the main chamber of the nozzle is not high enough to remove material, but when the slurry leaves the nozzle it accelerates to pass through the gap between the nozzle edge and the surface, see Figure 1. Due to this increased velocity, material removal occurs in a ring-shaped area, like in the case of FAUST [2],[3]. The nozzle is rotated to average out any irregularities in the nozzle. With a rotational speed of 1000 rotations per minute and an outside diameter of $16 \mathrm{~mm}$, the nozzle edge has a velocity of $0.8 \mathrm{~m} / \mathrm{s}$. 
This velocity is chosen to be small compared to the radial velocity of the water which is in the order of $20 \mathrm{~m} / \mathrm{s}$ (depending on the distance between the nozzle and the surface of course).

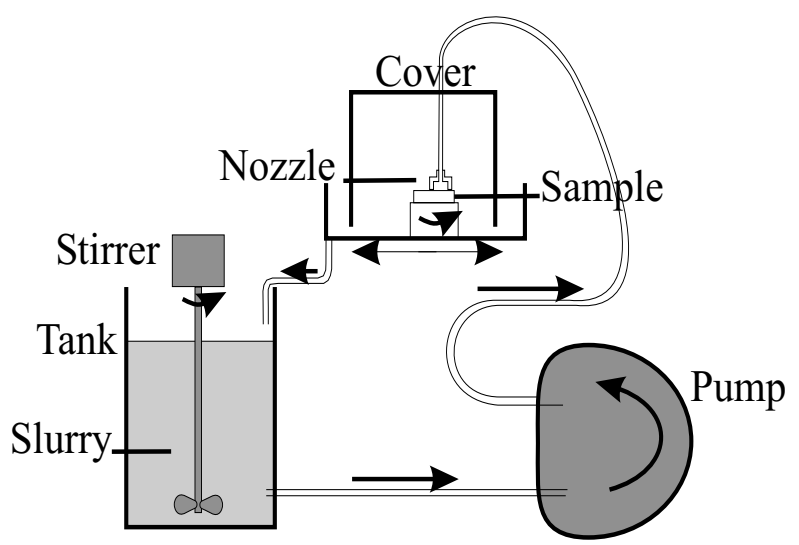

b

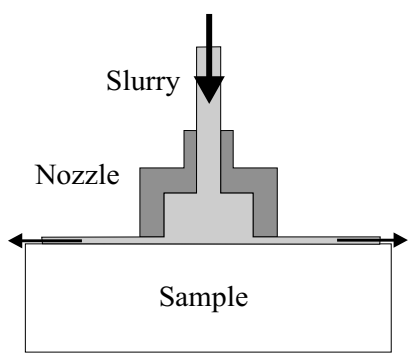

Figure 1: a) Schematic picture of the Jules Verne setup and b) a detail of the circularly symmetric nozzle. The slurry flows into the nozzle from above. The velocity is at its maximum where the nozzle and the surface are closest to each other. The slurry flows away in a radial direction after leaving the nozzle.

A wide variety of slurries, material to be machined, nozzle geometries and nozzle materials can be used for the processing. An advantage of using ceramic or sapphire nozzles is that they do not wear rapidly. An advantage of using plastic nozzles is the low risk when contact accidentally occurs between the nozzle and the surface. Since the material removal depends on the pressure build-up caused by the small distance between the nozzle and the workpiece, the process has an automatic maximum to the amount of removal for a stationary nozzle (feed-controlled).

A commercially available CNC optical fabrication machine has been modified at FISBA-Optik in such a way that both FJP and JV can operate on it, see Figure 2. The sample can be translated in one direction, and it can rotate. The nozzle can rotate and it can be positioned at a certain height and at an angle with respect to the sample surface.

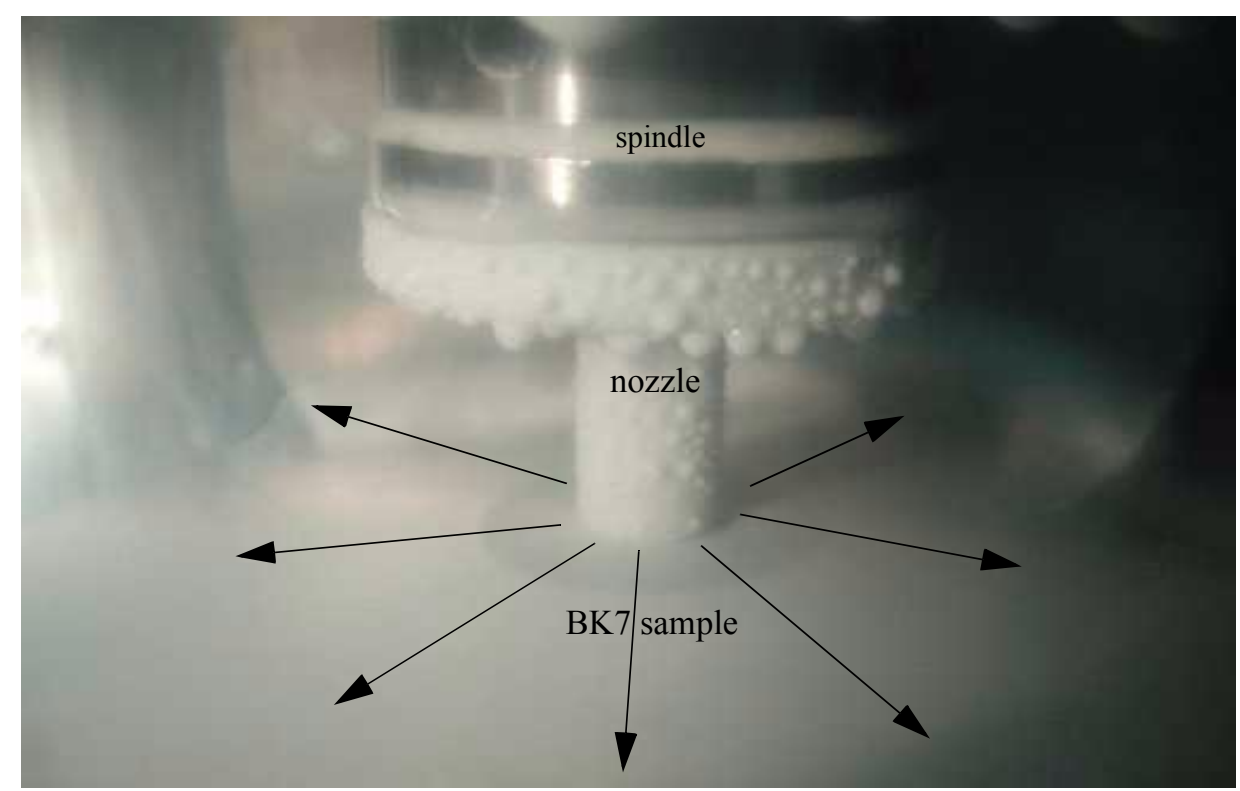

Figure 2: Detailed picture of the Teflon nozzle close to the sample, while processing a flat BK7 sample on the experimental Jules Verne setup on the modified CNC-machine at FISBA-Optik. The arrows indicate the flow direction of the slurry. 


\subsection{Principle of material removal}

An important property of a surface processing technique is its material removal per particle or per pass of the tool. This determines the speed of the process and it is responsible for the final surface state. A very coarse first division is the transition between ductile and brittle material removal. This transition is marked by the critical depth of cut, which is equal to $64 \mathrm{~nm}$ for BK7 glass [6]. This means that if more material is removed by one particle the removal will be in the brittle regime. If less material is removed it will be in the ductile regime [7]. The penetration depth of the particles depends on their velocity perpendicular to the surface. It has been shown that the material removal for FJP is in the ductile regime [7]. The material removal in the Jules Verne case will be in the ductile regime up to much higher velocities than in the case of FJP, since the particles in the JV case move over the surface at a much smaller angle. Their velocity perpendicular to the surface is much smaller.

A course estimate of the average velocity of the slurry can be obtained from the mass balance. The amount of water that flows into the nozzle should also flow out of the nozzle. The average slurry velocity will depend on the surface area through which the slurry can leave the nozzle. If we assume a constant flow of 10 liter per minute, an inner diameter of the nozzle of $6 \mathrm{~mm}$ and an outer diameter of $16 \mathrm{~mm}$ the velocity of the nozzle, infinitely far away from a surface, will be $5.9 \mathrm{~m} / \mathrm{s}$. When the diameter of that part of the nozzle where it comes closest to the surface is $10 \mathrm{~mm}$ the velocity for a gap distance $\Delta$ will be:

$$
v=\frac{1}{60 \pi}\left(\frac{1}{\Delta}\right)
$$

The dependence between the velocity and the gap distance is depicted in Figure 3.

velocity as a function of stand-off distance

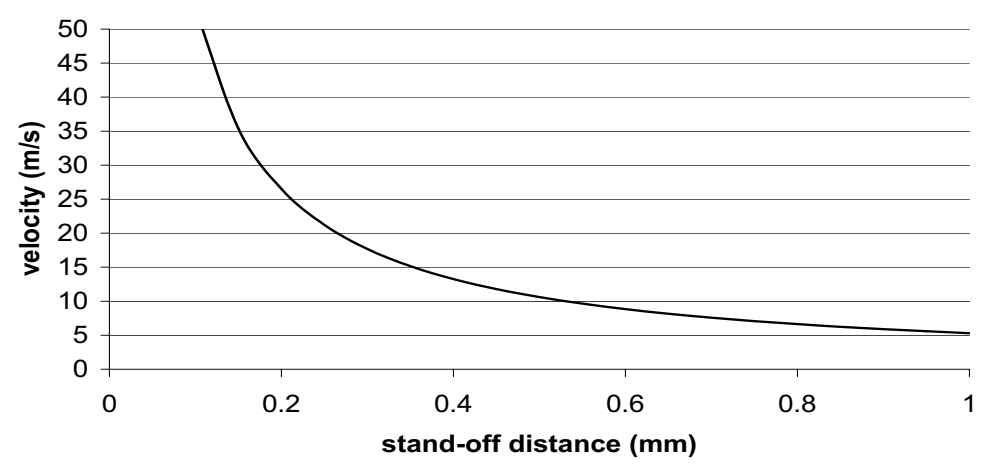

Figure 3: The theoretically occuring slurry velocity at the narrowest opening between the nozzle and the surface, as a function of the distance $\Delta$ to the surface, when assuming that the flow is circularly symmetrical.

\subsection{Advantages and disadvantages of the Jules Verne technique}

The JV technique has advantages and disadvantages with respect to FJP and with respect to other grinding and polishing techniques in general and we list them below.

Advantages:

- Nozzle diameter can be chosen freely (possibility of suppressing mid-spatial frequencies)

- Non-circular nozzles can be used (possibility of making aspheres).

- The type, concentration and diameter of abrasives in the slurry can be chosen.

- The $\mathrm{pH}$ value and viscosity of the slurry can be optimised to suit the process.

- The process is sensitive to stand-off distance. This means that a natural limit of removal depth exists. When the nozzle is close to the surface material is removed. This removed material causes the gap between the nozzle and the surface to increase, and this reduces the material removal speed. This makes it possible to remove e.g. diamond turning grooves, because the higher parts of the grooves are removed, while the lower parts can not be removed. A critical remark 
should be made here, since the gap is in the order of $50 \mu \mathrm{m}$, and the removal in the order of $1 \mu \mathrm{m}$, so the removal will not completely stop, but it will be slowed down further away from the nozzle.

- Better roughness should be obtainable than in the case of FJP owing to the fact that the slurry moves parallel to the surface in the radial direction.

\section{Disadvantages:}

- The $z$ controlling is more severe than in the case of FJP, because the distance to the surface should be controlled very accurately.

- Deep area's are not easily reachable, because the process is sensitive to stand-off distance.

- There is a limitation on the shapes that can be produced, depending on the diameter of the nozzle that is used. This can partially be overcome by combining the FJP and the JV technique. Deeper areas can be treated by FJP (higher pump flow) and better reachable areas can be treated in the JV mode.

\section{SIMULATION OF THE FLOW}

To describe the flow of the slurry and more specifically the movement of the abrasive particles in the Jules Verne (JV) case, a simulation has been carried out. To obtain information on the position and velocity of impact of the abrasive particles the following path is followed. The area of interest is defined and divided in small rectangular pieces, boundary conditions are imposed, the streamlines are computed over the entire area of interest, the velocity is computed from the streamlines, and particles are released in this flow. Each of these concepts will be explained below.

\subsection{Boundary conditions}

The flow in the nozzle was assumed to be according to the picture shown in Figure 4. The slurry flows into the nozzle from the top and flows away to the left and right hand side. Because the flow is symmetrical no slurry can flow across the center line, and the area of interest is thus limited to the grey area. These boundary conditions are used for a finite difference calculation that determines the position of all the streamlines. The definition of a streamline $\Psi$ is that it is a contour on the surface of the streamfunction. Streamlines are continuous lines on which the tangent of any point is parallel to the velocity at that point. The difference in height or value of two streamlines is related to the mass flux between the streamlines.

The value of the outermost streamlines is arbitrarily chosen to be zero at the sample (bottom) and on the center line (left) and since the stream function is per definition constant along a solid object it is arbitrarily chosen to have a value of $\Psi=$ 10 for the nozzle surface. This value determines the amount of mass flux through the area of interest. At the in- and outflow the stream function increases linearly from the value zero to ten, since the velocity is constant over the in and outflow openings.

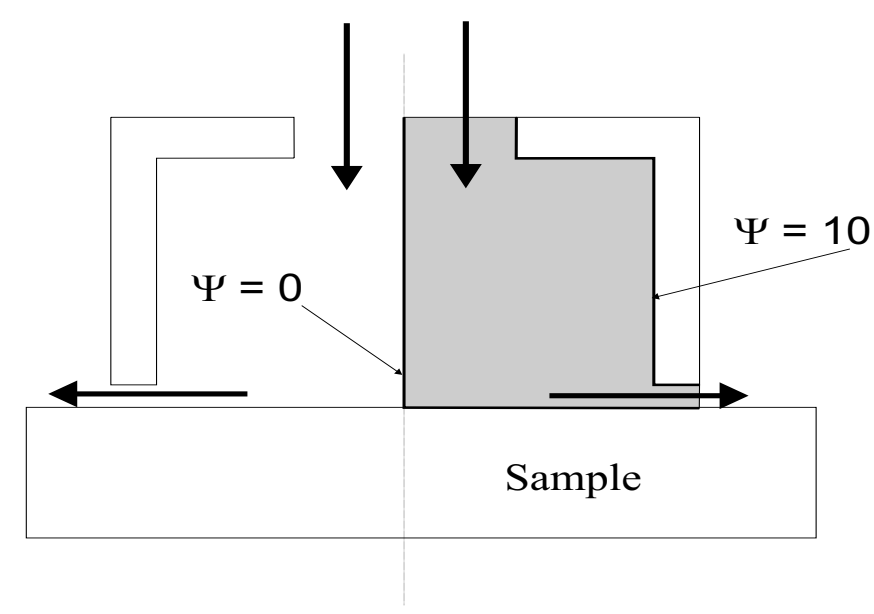

Figure 4: The nozzle geometry with the area of interest highlighted in grey. The slurry flows into the area of interest from above, and it flows away to the sides. The value of the stream function $\Psi$ is given for the boundaries. 


\subsection{Computation of intermediate streamlines}

To compute the position of the intermediate streamlines a finite-difference program is used according to the following procedure [4]. The stream-function $\psi$ has to conform to Laplace's equation:

$$
\frac{\partial^{2} \psi}{\partial x^{2}}+\frac{\partial^{2} \psi}{\partial y^{2}}=0
$$

The function $\psi$ is defined at discrete positions $(i, j)$ only, this will be denoted as $\psi_{i, j}$, see Figure 5 . The algebraic approximations of the first and second derivatives of $\psi$ in the $x$ and $y$ directions are:

$$
\begin{aligned}
& \frac{\partial \psi}{\partial x} \approx \frac{1}{\Delta x}\left(\psi_{i+1, j}-\psi_{i, j}\right) \\
& \frac{\partial \psi}{\partial y} \approx \frac{1}{\Delta y}\left(\psi_{i, j+1}-\psi_{i, j}\right) \\
& \frac{\partial^{2} \psi}{\partial x^{2}} \approx \frac{1}{\Delta x^{2}}\left(\psi_{i+1, j}-2 \psi_{i, j}+\psi_{i-1, j}\right) \\
& \frac{\partial^{2} \psi}{\partial y^{2}} \approx \frac{1}{\Delta y^{2}}\left(\psi_{i, j+1}-2 \psi_{i, j}+\psi_{i, j-1}\right)
\end{aligned}
$$

When these approximations are combined with Laplace's equation we find:

$$
\psi_{i, j} \approx \frac{1}{4}\left(\psi_{i, j+1}+\psi_{i, j-1}+\psi_{i+1, j}+\psi_{i-1, j}\right)
$$

The stream-function in each central point thus equals the average of the four neighboring stream-function values. The numerical error of the discretised equation as compared to the exact solution of Laplace's equation is proportional to the square of the mesh size $\Delta x$ and $\Delta y[4]$.

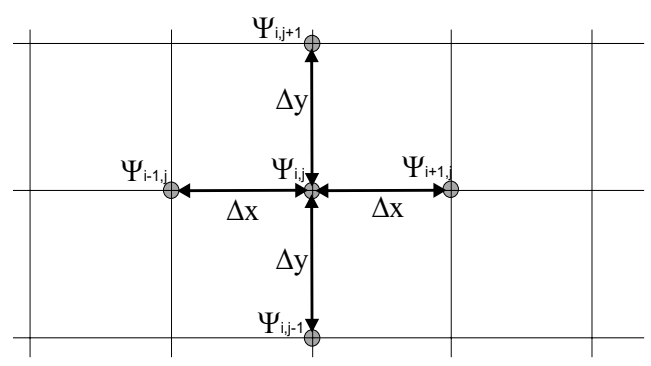

Figure 5: Overview of the stream-function $\Psi$ at a point $(i, j)$ and at its four neighboring points.

A routine has been written in Matlab that computes the intermediate values according to Equation (4). As input the boundary conditions and the grid are given. After a few hundred iterations the stream-function is known in all intermediate points. See for example the streamline profile shown in Figure 6 a).

\subsection{Velocity calculation}

The velocity of the flow can be computed at any point from the stream-function, according to White [4] by using:

$$
\begin{aligned}
& v_{x}(i, j)=\frac{\psi_{i, j+1}-\psi_{i, j}}{\Delta y} \\
& v_{y}(i, j)=-\frac{\Psi_{i+1, j}-\psi_{i, j}}{\Delta x}
\end{aligned}
$$


The final step is to compute where, under which angle, and with which velocity, particles impact the surface. This depends on where the particles are released in the flow that is characterised by the velocity distribution as computed from the stream-function using Equation (5).

\subsection{Single particle trajectory}

In his $\mathrm{PhD}$ thesis, van Haarlem [5] describes the force law that particles have to obey, in the case that the density of the particles is much larger than that of the fluid. The force on a single particle $F$ depends on the mass of the particle $m_{p}$, the velocity of the fluid $v_{f}$ and that of the particle $v_{p}$, the gravitational acceleration $g$, and the particle response time $\tau_{p}$ according to:

$$
F=\frac{m_{p}}{\tau_{p}}\left(v_{f}-v_{p}\right)+m_{p} g
$$

This response time can be seen as a time scale over which the particles respond to changes in the flow conditions. The particle response time is defined as:

$$
\tau_{p}=\frac{2 \rho_{f}\left(\frac{d}{2}\right)^{2}}{9 \rho_{p} \eta_{f}} .
$$

where $\rho_{f}$ is the density of the fluid $\left(\mathrm{kg} / \mathrm{m}^{3}\right), \rho_{p}$ the density of the particle $\left(\mathrm{kg} / \mathrm{m}^{3}\right), d$ the abrasive particle diameter, and $\eta_{f}$ the kinematic viscosity of the fluid.

As initial condition the particle can be assumed to have the same velocity as the fluid. The force on the particle is computed, the resulting displacement of the particle in the fluid is computed, and at the new position of the particle this process is repeated. After many iterations the total trajectory of the particle is known. From the two equations above one can see that small particles or particles in a viscous fluid will follow the velocity distribution of the fluid very closely. Larger or heavier particles or particles in a less viscous flow will deviate more from the equi-velocity lines in the fluid.

The equations (6) and (7) do not take into account what happens when the particles would leave the area of interest. In stead of making them bounce off the walls we decided to let them continue through them. Outside of the flow their velocity automatically decreases because the fluid velocity is zero. An example of the computed particle trajectories is given in Figure $6 \mathrm{~b}$ ). The simulation shows that most particles make contact with the surface there where the slurry leaves the nozzle, and where the velocity is the highest. This simulation approach could be used to compare the effect of different geometries of the nozzle wall.

a

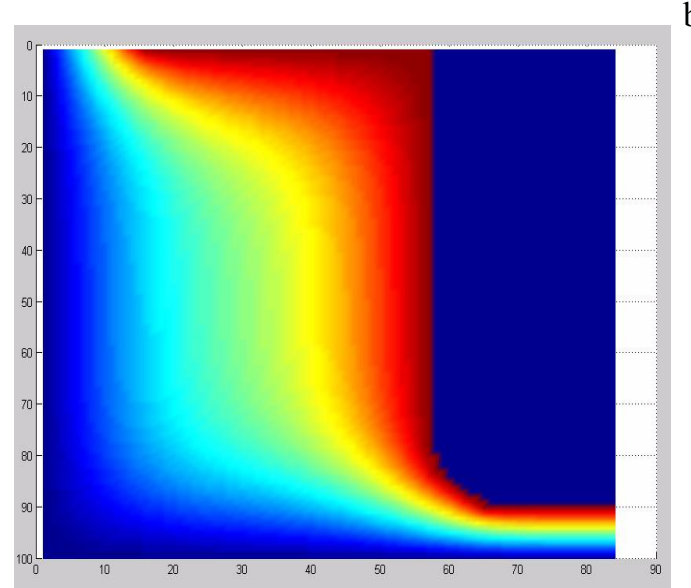

b

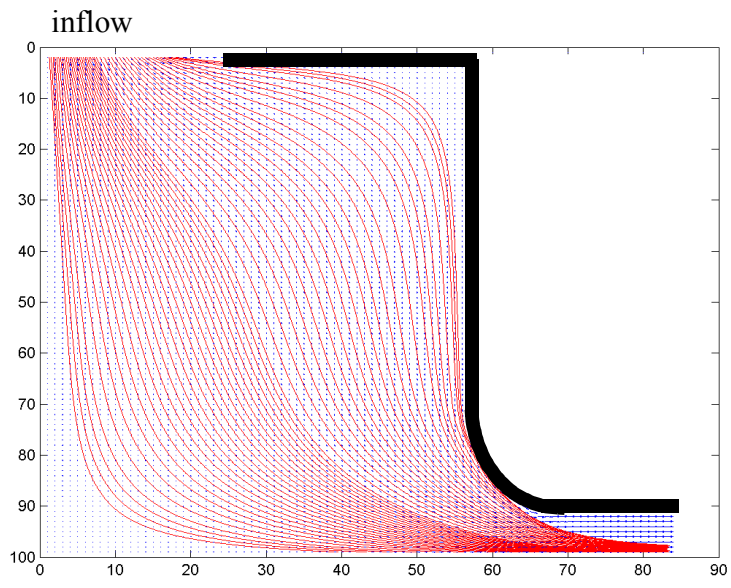

Figure 6: a) The flow profile. b) The trajectory that particles with a certain mass would follow in this flow. In the simulation all particles are released in the inflow opening at the top of the picture. More particles are released in the left half of the opening, since these particles reach the surface, and those that start at the right half of the opening do not. This is not easily seen from this small figure, but it follows from the particle trajectories. 


\section{INITIAL EXPERIMENTS WITH JV}

\subsection{Proof of principle}

As a proof of the JV principle, the pressure in the hose leading to the nozzle has been measured as a function of the distance between the nozzle and the surface for a number of different settings of the pumps pressure. The pressure indeed increases when the distance gets smaller, and higher pressures can be reached if the pump power is increased, as can be seen in Figure 7.

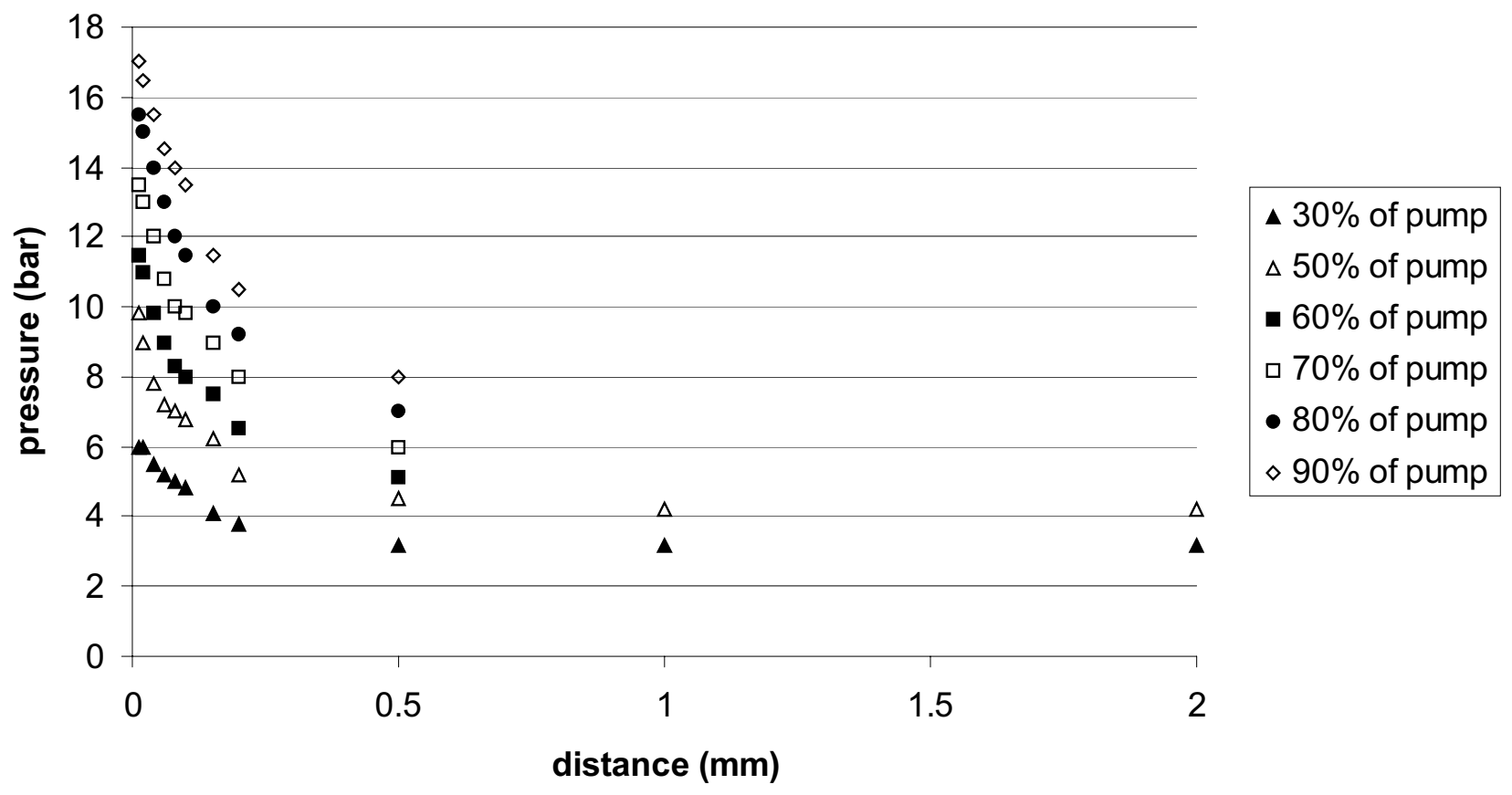

Figure 7: Pressure as a function of the distance between the nozzle and the surface at $30,50,60,70,80$, and $90 \%$ of the pump power.

\subsection{Stationary spot}

The next experiment was to remove material from one location on a flat, pre-polished $40 \mathrm{~mm}$ diameter BK7 sample. The sample did not rotate or translate, but the nozzle did rotate in order to average out any inhomogeneities in the nozzle's edge. The nozzle that was used had an outer diameter of $16 \mathrm{~mm}$ and an inner diameter of $6 \mathrm{~mm}$. A cross-section of the nozzle can be found in Figure $8 \mathrm{a}$ ). The diameter of the ring that comes in closest contact with the surface is $11 \mathrm{~mm}$. The resulting surface (after 30 minutes of processing) on the initially polished surface can be seen in Figure 8 b). In the ring shaped area where the nozzle and the workpiece were closest together most material has been removed (approximately $380 \mathrm{~nm}$ ). In the center no material has been removed and in the area further away from the center some material has been removed. 


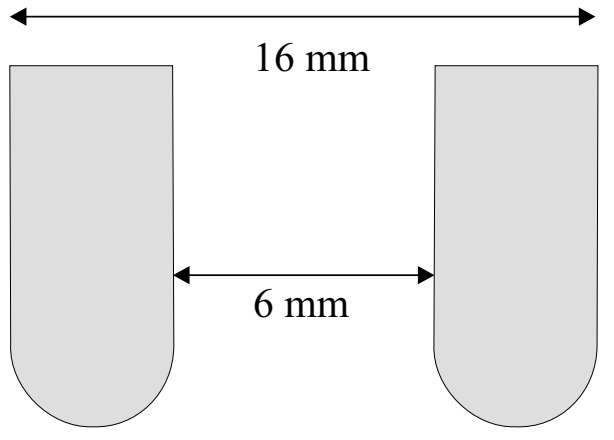

$\mathrm{b}$

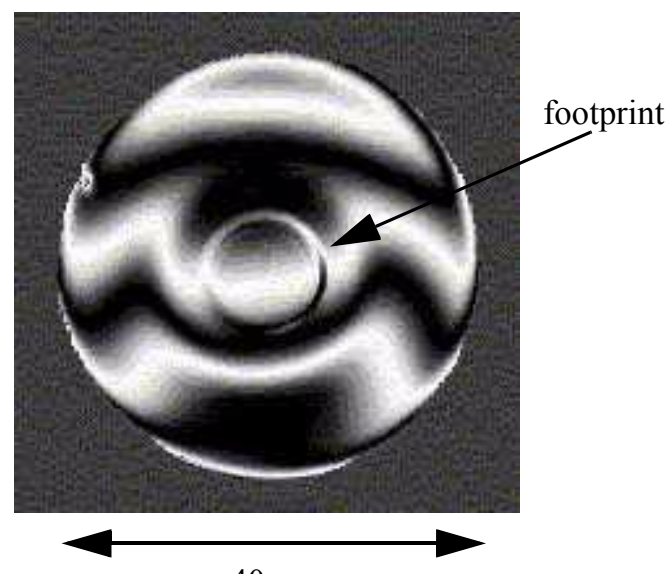

$40 \mathrm{~mm}$

Figure 8: a) Cross-section through the nozzle that was used, with dimensions indicated. b) Interferogram of the footprint that has been created by the JV nozzle shown in a) in the stationary mode. The wavelength of the light that was used is $\lambda=632.8 \mathrm{~nm}$.

\section{ROUGHNESS}

Since the Jules Verne technique is a limiting case of the FJP technique, and more experiments have been done with FJP, this section will describe some considerations on the roughness from the FJP point of view. The fluid jet polishing (FJP) setup has been described before by the authors [7]. Recently a better understanding of the final surface roughness has been obtained. Experiments have shown that with the FJP technique it is possible to reduce the surface roughness from an initially high value, but when starting on a polished surface the roughness always increases somewhat. This does not occur in classical polishing. The difference between FJP and classical polishing is the averaging effect that occurs for classical polishing versus the random nature of the individual impacts in the case of FJP. First the experimental observations of the changes in the roughness of a surface will be described, then a model will be described to explain the observed effects.

\subsection{Experimental investigation of the roughness}

To investigate the influence of the FJP process on the roughness of a surface as a function of time, and to see if it depends on the initial roughness, the following experiment has been carried out. With a 5\% \#1200 SiC slurry three different samples were treated. The first sample was polished (average roughness $R_{a}<1 \mathrm{~nm}$ ) the second was pre-ground with $\# 800 \mathrm{SiC}\left(R_{a}=400 \mathrm{~nm}\right)$, and the third was pre-ground with $\mathrm{SiC} \# 400\left(R_{a}=600 \mathrm{~nm}\right)$. The amount of removed material and the roughness of the samples was measured after 1,2, 5, 10,30, and 60 minutes. The resulting graphs can be seen in Figure 9. From these experiments one can conclude that the material removal does depend on the initial roughness. The material removal is the highest for the roughest surfaces. This can be explained from the fact that abrasive particles in the flow that move parallel to the surface can more easily remove material from a very hilly surface with protruding peaks than from a perfectly flat surface. Particles that impact perpendicularly on the surface will not notice any difference. It could also be explained from the fact that the rough surface causes more turbulence in the flow, which increases the material removal rate [8]. The roughness of both the rough surfaces decrease as a function of time, while the roughness of the smooth surface increases. 

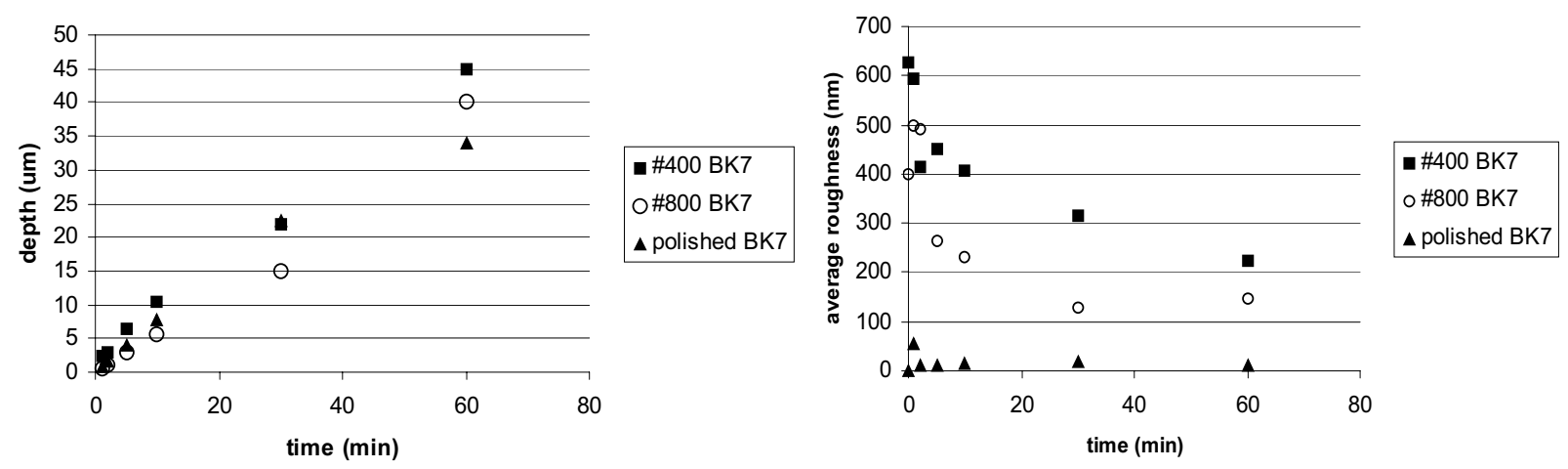

Figure 9: a) Depth and b) roughness as a function of time for three surfaces with different initial roughness.

To investigate if the final surface roughness depends on the initial surface roughness the following experiment has been conducted. From an \#800 SiC ground BK7 surface with an initial average roughness of $300 \mathrm{~nm}, 20.4 \mu \mathrm{m}$ was removed with a $10 \% \# 800 \mathrm{SiC}$ slurry. This resulted in a surface with $R_{a}=6.4 \mathrm{~nm}, R_{q}=8.3 \mathrm{~nm}, R_{z}=60.3 \mathrm{~nm}$, and $R_{t}=76.0 \mathrm{~nm}$. From a polished surface with an initial roughness of $R_{a}=1 \mathrm{~nm}, 19 \mu \mathrm{m}$ was removed, which resulted in a surface with $R_{a}$ $=6.0 \mathrm{~nm}, R_{q}=7.8 \mathrm{~nm}, R_{z}=61.2 \mathrm{~nm}$, and $R_{t}=89.6 \mathrm{~nm}$. The Wyko roughness measurements of both samples can be found in Figure 10. We can thus conclude that the final roughness of a surface does not depend on the initial roughness. Other experiments have shown that when only $350 \mathrm{~nm}$ of material is removed from an initially polished surface, the roughness only increases from approximately $R_{a}=1 \mathrm{~nm}$ and $R_{z}=7 \mathrm{~nm}$ to $R_{a}=1.5 \mathrm{~nm}$ and $R_{z}=15.6 \mathrm{~nm}$. A removal of $350 \mathrm{~nm}$ would be sufficient to make shape corrections that improve a " $\lambda / 2$ " surface to the correction level of $\lambda / 10$ or better.
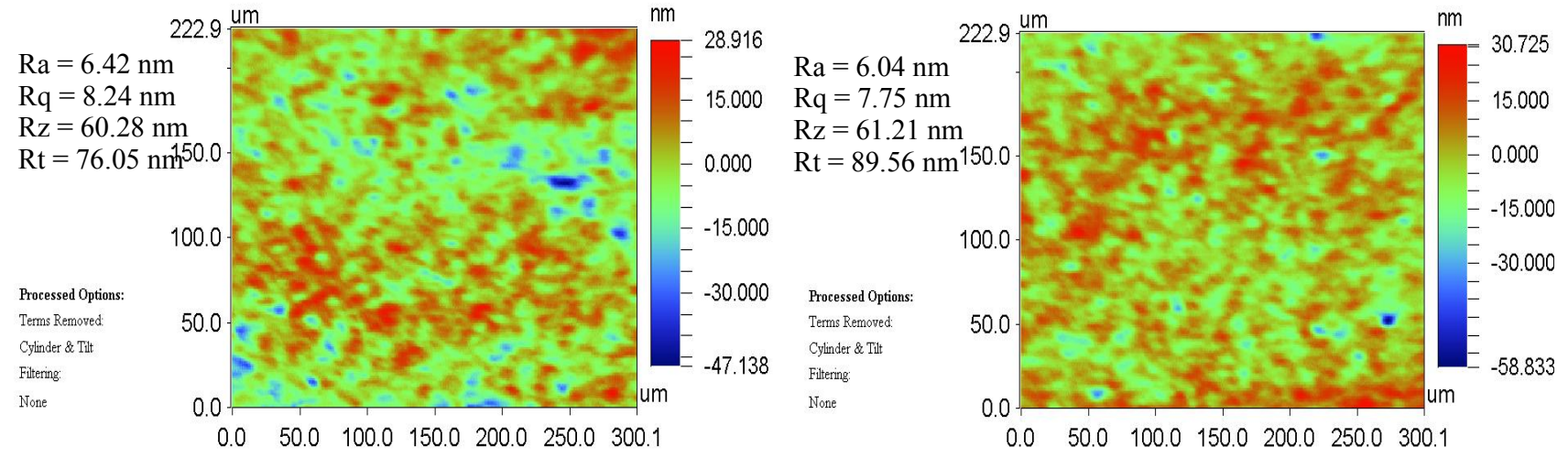

Figure 10: Comparison of surface structure and roughness values for two samples that were treated identically, but had a different initial surface roughness. Wyko measurements of the final states of a) the initially ground surface and b) the initially polished surface.

\subsection{Simulation of the roughness}

The roughness that results during the processing is an important issue. When starting on a polished surface one wants to remove material without increasing the roughness, and when the initial surface roughness is high, one wants to decrease the rms roughness to a few nanometers for low quality optics and to less than $1 \mathrm{~nm}$ for higher quality optics. To investigate which parameters determine the final roughness a model has been developed. The simulated resulting roughness will be described here. The experimentally obtained roughness results have been described above. 
Surface roughness is calculated from the height distribution of a surface. The change in the height profile of a surface is caused by the material removal of single impacting particles. In our model particles are assumed to impact at random locations on the surface. When it is not possible for the particle to impact the random location, due to protruding areas around this location, then the material removal that should occur in the center is distributed evenly over all points of contact between the particle and the surface. Assumptions have been made that all impacting particles have an identical mass, velocity, angle of impact, and each removes the same amount of material when impacting. The effects of the shape of the particles, their hardness, and their strength are not taken into account.

As the surface upon which particles can impact 10 x 10 elements are chosen. In Figure 11 a) the roughness of an initially perfectly smooth surface has been plotted as a function of the number of impacts (from 0 to 10000). In Figure 11 b) the same number of impacts occurred on a surface that initially had a random height distribution between 0 and 100 . In Figure $11 \mathrm{c}$ ) the initial surface has a random distribution between 0 and 1000. The roughness of the smooth surface increases to a certain value. The roughness then fluctuates around the equilibrium value. The roughness of the rougher surface decreases to a certain value and then remains stable there. The roughness of the very rough surface decreases, but does not reach the same low roughness as the other surfaces within the first 10000 impacts. The final roughness is not reached, because the average number of impacts per element is only 100, and the initial height difference between two neighboring points is maximally 1000 . When the impacts continue over a longer time period (approximately 50000 impacts) the same final roughness will be reached as in the case of the less rough initial surface.

Other parameters that can be varied within this model are the effect of the impact of a single particle and the diameter of the impacting particle. When the effect of one particle is increased (which can be compared to increasing the pressure or the energy of the particle) the roughness of rough surfaces decreases more rapidly, but the finally obtained roughness is also higher than it would be for lower pressures. This can be explained since each particle has a larger effect, so on a smooth surface the impact of one particle will increase the roughness more than for a particle at lower pressures. When the diameter of the impacting particle is increased (without increasing its impacting energy) the roughness decreases at the same rate as with small particles, but the final surface roughness is better for larger particles, because these particles have an effect over an area that is larger, so the removal will be more uniform.

From these simulations one can see that the best roughness will be obtained for large abrasive particles, with a low kinetic energy (small mass and small velocity perpendicular to the surface). In the limiting case this comes down to the setup used for classical polishing.

The viscosity of the fluid and the angle between the nozzle and the surface can both be used as a parameter to reduce the velocity component of the particles perpendicular to the surface. The advantage of changing the viscosity is that the area over which the material is removed is not changed as drastically as in the case when the impact angle is changed. 
a

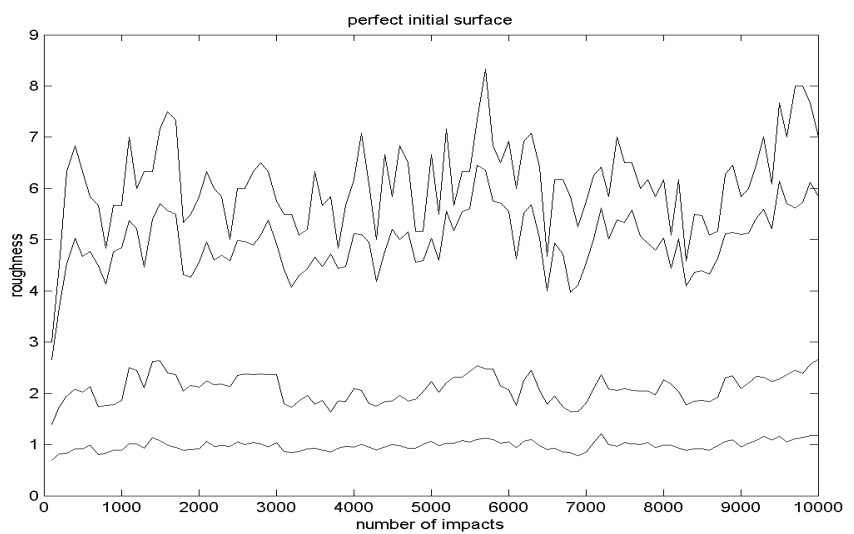

b
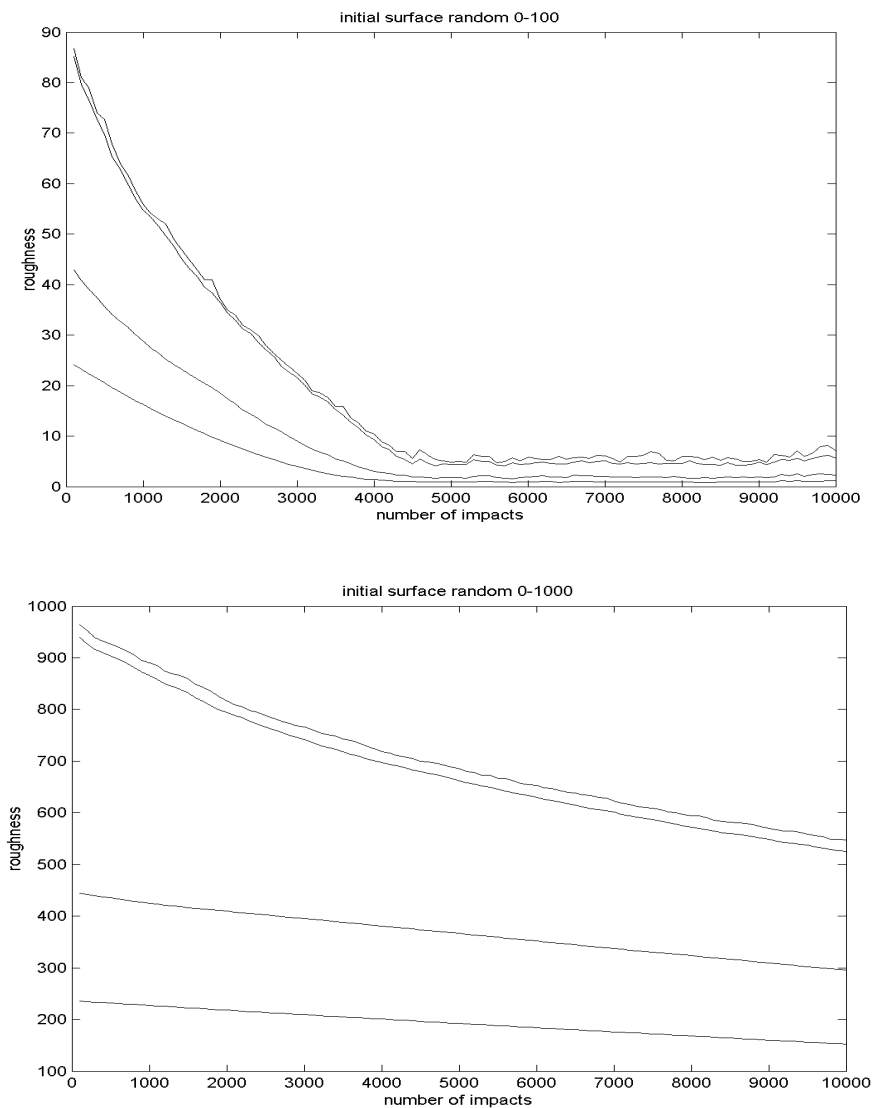

Figure 11: Roughness values as a function of the number of impacts. Each figure shows the $R_{a}, R_{q}, R_{z}$ and $R_{t}$ value (from the bottom to the top curve) in arbitrary units. a) shows an initially smooth surface, b) shows an initial surface with randomly distributed height values between 0 and 100, and c) shows an initial surface with randomly distributed height values between 0 and 1000.

\section{CONCLUSIONS}

The Jules Verne (JV) method is an interesting variation on the FJP process. An abrasive slurry is fed into the cup wheel along its axis of symmetry. If the gap between tool and workpiece is small enough, pressure is built up within the cup wheel and material removal is achieved along the circular edge of the rotating cup wheel. This principle enables a feed- 
controlled machining process. A high speed polishing machine has been modified at FISBA-Optik in such a way that both FJP and JV can operate on it. With the type of slurry, the kind of nozzle and the velocity of the flow that is used in our setup the material removal is in the ductile regime. An advantage of the JV technique is that the slurry moves very close to parallel over the surface, which should result in better surface roughness values than in the case of FJP. A disadvantage of the JV technique is that there is a limitation to the shapes that can be produced, depending on the diameter of the nozzle.

A simulation of the fluid flow and of the expected trajectories of the abrasives has been carried out. The simulation shows that most particles make contact with the surface there where the slurry leaves the nozzle, and where the velocity is highest.

Some initial experiments have been carried out with JV that show how the pressure is related to the stand-off distance, and the experiments show that material removal is indeed possible with the JV technique.

Some attention has been given to the study of roughness as well. Material removal and roughness have been monitored in the coarse of an FJP experiment for different initial surface roughness values. The material removal can be seen to be higher for rougher surfaces. The final roughness is independent of the initial roughness though. Finally, a simulation has been presented that predicts the surface roughness for a surface with an initial roughness that is impacted at random locations by abrasive particles.

\section{REFERENCES}

[1] Oliver W. Fähnle, Hedser van Brug, and Hans J. Frankena, 'Fluid jet polishing of optical surfaces,' Appl. Opt., 37 (28), pp.6771-6773 (1998)

[2] O.W. Fähnle, H. van Brug, C.J. van der Laan, and H.J. Frankena, 'Loose abrasive line-contact machining of aspherical optical surfaces of revolution,' Appl. Opt., 36 (19), pp.4483-4489 (1997)

[3] Oliver W. Fähnle, 'Shaping and finishing of aspherical optical surfaces,' PhD thesis, TU Delft (1998)

[4] Frank M. White, 'Fluid Mechanics,' WCB/ McGraw-Hill (1999)

[5] Bas Adriaan van Haarlem, 'The dynamics of particles and droplets in atmospheric turbulence, a numerical study,' PhD thesis, Delft University of Technology (2000)

[6] T.G. Bifano, T.A. Dow, and R.O. Scattergood, 'Ductile regime grinding: a new technology for machining brittle materials,' J. of Eng. for Ind., Trans. of the ASME, 113, pp.184-189 (1991)

[7] Silvia M. Booij, Hedser van Brug, Joseph J.M. Braat, Oliver W. Fähnle, 'Nanometer deep shaping with fluid jet polishing,' Opt. Eng., 4, pp. 1926-1931, (Aug. 2002)

[8] I. Finnie, 'Erosion of surfaces by solid particles,' Wear, 3, pp.87-103 (1960) 